\title{
Inverse Kramers formula and fusion dynamics of heavy ions
}

David Boilley ${ }^{1}$, Yasuhisa $\mathrm{Abe}^{2}$, and Jing-Dong $\mathrm{Bao}^{3}$

${ }^{1}$ GANIL, BP 55027, F-14076 Caen cedex, France, e-mail: boilley@ganil.fr

2 Yukawa Institute for Theoretical Physics, Kyoto University, Kyoto 606-8502, Japan

${ }^{3}$ Department of Physics, Beijing Normal University, Beijing 100875, China

To be published in Eur. Phys. J. A

\begin{abstract}
Fusion probability of colliding heavy ions is evaluated by exactly solving a Langevin equation with a parabolic potential barrier. The model, which accomodates dissipation in the approaching phase, leads to an inverse Kramers's formula and an expression of the extra-push energy. In the very specific case of an overdamped regime, an Arrhenius type formula is obtained.
\end{abstract}

PACS. 02.50.Ey Stochastic processes - 05.40.Jc Brownian motion - 25.70.Jj Fusion and fusion-fission reactions

Superheavy elements are predicted to exist due to shell closures of nucleons giving an extra stability. Heavy-ion reactions are used for their synthesis and it is both an experimental and theoretical challenge due to very low production probabilities. For recent reviews, see Refs. [1, 2]. In order to predict residue cross sections of the superheavy elements, we firstly need to understand mechanisms of fusion reactions. However, fusion mechanisms of massive nuclear systems are not yet well understood, though it is known that fusion is hindered in systems with $Z_{p} * Z_{t}$ larger than 1,600 , where $Z_{p}$ and $Z_{t}$ are atomic numbers of projectile and target nuclei: systems do not fuse even with incident energies being well above the expected Coulomb barrier. So-called extra-push energy is necessary in addition to the barrier height. This could be interpreted by a friction force acting between two colliding nuclei in the approaching phase as well as by dissipation of energy of a collective motion which leads the amalgamated system to a spherical compound nucleus. It is most likely that both mechanisms exist, though which one is dominating in which situation is not yet known. Thus, fusion could be divided into two steps, and then fusion probability is given by the product of two factors, i.e., the sticking probability for the colliding system to stick each other into an 
amalgamated system and the formation probability of the spherical compound nucleus, starting from the pear-shape configuration made of the colliding nuclei $[3,4]$. These two processes are not independent of each other. In the present note we focus our study on the latter one.

As is well known, there is a conditional saddle point or a ridge line between the pear-shaped configuration and the spherical shape on the energy surface of the Liquid Drop Model, which is to be overcome for the system to form a compound nucleus. For hot fusion systems, a fluctuationdissipation dynamics has been used by various authors to study the process [4-9]. The underlying assumption in applying a thermal transport equation to such a process relies on the fact that internal degrees of freedom are fastly thermalized compared to the time scale of the collective degrees of freedom. If in addition, dissipation already occurs in the approaching phase as in the present model, the heating-up process is mostly completed and the use of a Langevin type equation is meaningful. The fluctuations due to the random force play a crucial role here, where the probabilities are very small. In nuclear physics, such a formalism has been successful to study fission [10,11], following Kramers's pioneering work [12]. Here, we are dealing with an 'inverse process' to fission of the compound nucleus.

Various assumption were done to solve the problem. First, as this model is limited to the so-called hot fusion mechanisms, a classical formalism would be sufficient. Quantum fluctuations were shown to have a small effects on fusion probability of hot nuclei [13]. Markovian ap- proximation, which is generally used, might appear as a strong assumption for such finite size systems. Studies of the importance of the memory kernel will be published elsewhere [14]. Other approximations like an overdamped regime were used in Refs $[8,9]$ without justification. The analytical solution proposed in Ref. [15] should help to clarify the dynamical process and compare the various approaches and approximations.

The analytical solution of the formation probability obtained in the multi-dimensional model [15] suggests the existence of an effective one-dimensional description. We, thus, investigate here a simplified one-dimensional model with a parabolic barrier and dissipation-fluctuation dynamics along the reaction coordinate. As will be seen later, even with this simplification, we can learn characteristic features of the inverse process, i.e., fusion mechanisms of massive systems. We believe that it has some interests in other fields of physics such as collision of molecular systems, atomic clusters, etc...

A Langevin equation is used for the collective degree of freedom passing into a potential well. The barrier is supposed to be an inverted parabola around the saddle point, $V(q)=-m \omega^{2} q^{2} / 2$ and the coefficients are supposed to be constant around the saddle. The Langevin equation reads,

$$
\ddot{q}+\beta \dot{q}-\omega^{2} q=r(t)
$$

where $r(t)$ comes from a Gaussian random force and satisfies the fluctuation-dissipation theorem,

$$
\langle r(t)\rangle=0 \quad \text { and } \quad\left\langle r(t) r\left(t^{\prime}\right)\right\rangle=\frac{2 T \beta}{m} \delta\left(t-t^{\prime}\right) .
$$


In these equations, $T, m$ and $\beta$ denote the temperature, the mass and the reduced friction, respectively. Given a set of initial conditions, $q_{0}<0$ and $p_{0}=m \dot{q}_{0}>0$, the Langevin equation can be exactly solved, see Refs. [15-17], and leads necessarily to a Gaussian distribution. Then, the probability at a given time that the particle has passed over the barrier at $q=0$ is simply,

$$
\begin{aligned}
P\left(t ; q_{0}, p_{0}\right) & =\int_{0}^{+\infty} \frac{d q}{\sqrt{2 \pi}} \frac{1}{\sigma_{q}(t)} \exp \left(-\frac{(q-\langle q(t)\rangle)^{2}}{2 \sigma_{q}^{2}(t)}\right) \\
& =\frac{1}{2} \operatorname{erfc}\left(-\frac{\langle q(t)\rangle}{\sqrt{2} \sigma_{q}(t)}\right)
\end{aligned}
$$

where the average trajectory reads,

$$
\langle q(t)\rangle=q_{0} R(t)+p_{0} S(t)
$$

with

$$
\begin{aligned}
R(t)= & e^{-x \omega t}\left[\cosh \left(\sqrt{1+x^{2}} \omega t\right)\right. \\
& \left.+\frac{x}{\sqrt{1+x^{2}}} \sinh \left(\sqrt{1+x^{2}} \omega t\right)\right] \\
S(t)= & \frac{1}{m \omega \sqrt{1+x^{2}}} e^{-x \omega t} \sinh \left(\sqrt{1+x^{2}} \omega t\right),
\end{aligned}
$$

and the variance,

$$
\begin{aligned}
\sigma_{q}^{2}(t)= & \frac{T}{m \omega^{2}}\left[e ^ { - 2 x \omega t } \left(2 \frac{x^{2}}{1+x^{2}} \sinh ^{2}\left(\sqrt{1+x^{2}} \omega t\right)\right.\right. \\
& \left.\left.+\frac{x}{\sqrt{1+x^{2}}} \sinh \left(2 \sqrt{1+x^{2}} \omega t\right)+1\right)-1\right]
\end{aligned}
$$

In the above equations, $x=\beta / 2 \omega$.

In the case of Kramers's problem [12], particles are thermally equilibrated in a potential well that has a small leak and indeed, Kramers's solution cannot be applied to the fusion problem as in Ref. [7]. In the inverse mechanism, the system is not equilibrated, at least with respect to a collective degree of freedom such as reaction coordinate. In the case of heavy ions fusion, dissipation already occurs in the approaching phase, generating a dispersion of the initial conditions, $q_{0}$ and $p_{0}[3]$. Subsequently, once the nuclei are in contact, a very strong dissipation is known to start up. Internal degrees of freedom accommodate the dissipated energy and are supposed to be quickly equilibrated at temperature $T$. Not the collective ones, of course. They are coupled to the intrinsic ones through the dissipationfluctuation terms. As a consequence, in such a situation, a dispersion of the initial conditions should be considered with a different width, assuming a Gaussian distribution $[3]$

$$
\begin{aligned}
W_{0}\left(\bar{q}_{0}, \sigma_{q_{0}}, \bar{p}_{0}, T_{0}\right)= & \frac{1}{2 \pi \sigma_{q_{0}} \sqrt{m T_{0}}} \exp \left[-\frac{\left(q_{0}-\bar{q}_{0}\right)^{2}}{2 \sigma_{q_{0}}^{2}}\right] \\
& \cdot \exp \left[-\frac{\left(p_{0}-\bar{p}_{0}\right)^{2}}{2 m T_{0}}\right]
\end{aligned}
$$

The new fusion probability can be easily calculated and becomes

$$
\begin{aligned}
\bar{P}\left(t ; \bar{q}_{0}, \sigma_{q_{0}}, \bar{p}_{0}, T_{0}\right) & =\int_{-\infty}^{+\infty} d q_{0} \int_{-\infty}^{+\infty} d p_{0} P \cdot W_{0} \\
& =\frac{1}{2} \operatorname{erfc}\left(-\frac{\langle\bar{q}(t)\rangle}{\sqrt{2} \sigma_{q}^{\prime}(t)}\right),
\end{aligned}
$$

where $\langle\bar{q}(t)\rangle$ is the same as in Eq. (4) provided that $q_{0}$ and $p_{0}$ are replaced by $\bar{q}_{0}$ and $\bar{p}_{0}$, respectively. The variance is larger,

$$
\sigma_{q}^{\prime 2}(t)=\sigma_{q}^{2}(t)+\sigma_{q_{0}}^{2} R^{2}(t)+m T_{0} S^{2}(t)
$$

In these equations, $T_{0}$ is a parameter for the initial distribution that could be interpretated as a temperature of the nuclei at contact while $T$ would correspond to the temperature of the amalgamated systems when the kinetic energy is dissipated. $T_{0}$ could be equal to the temperature $T$ of the Langevin force in the case of a complete damping of the initial kinetic energy, i.e. $\bar{p}_{0}=0$. 
For large times, $\left(\sqrt{1+x^{2}}-x\right) \omega t \gg 1$, the probability to pass over the barrier converges to a finite value,

$$
\begin{aligned}
& \bar{P}\left(t \rightarrow+\infty ; \bar{q}_{0}, \sigma_{q_{0}}, \bar{p}_{0}, T_{0}\right) \\
& \rightarrow \frac{1}{2} \operatorname{erfc}\left(\sqrt{\frac{\bar{B}}{T^{\prime}}}-\frac{1}{\left(x+\sqrt{1+x^{2}}\right)} \sqrt{\frac{\bar{K}}{T^{\prime}}}\right),
\end{aligned}
$$

where $\bar{K}=\bar{p}_{0}^{2} / 2 m$ denotes the average initial kinetic energy, $\bar{B}=m \omega^{2} \bar{q}_{0}^{2} / 2$ the average barrier height and

$$
T^{\prime}=T_{0}\left(\sqrt{1+x^{2}}-x\right)^{2}+m \omega^{2} \sigma_{q_{0}}^{2}+2 T x\left(\sqrt{1+x^{2}}-x\right),
$$

a "dynamical temperature". This result is coherent with the one of Ref. [18] derived in a different context. In the case of Kramers's problem, temperature is solely responsible for the diffusion because of the initial equilibrated distribution inside the pocket. Here, there is an interplay between dynamics and diffusion: the initial kinetic energy should also be taken into account and the "temperature" $T^{\prime}$ depends on initial conditions. For a sharp initial condition, $T_{0}=0$ and $\sigma_{q_{0}}=0$, this result is similar to the one of Refs. [15,19].

To have half of the particles to pass over the barrier, the initial kinetic energy should be

$$
\bar{K}=\left(x+\sqrt{1+x^{2}}\right)^{2} \bar{B} .
$$

This means that the average trajectory has to overcome an effective barrier, $B_{e f f}=\left(x+\sqrt{1+x^{2}}\right)^{2} B$ that takes viscosity into account. In the very weak friction limit, the above condition becomes $B_{\text {eff }} \simeq B$, which is a trivial result. Taking usual values in nuclear physics, $\hbar \omega=1 \mathrm{MeV}$ and $\beta=5.10^{21} s^{-1}, B_{e f f} \simeq 10 B$. This shows the important role played by dissipation which is expected to be responsible for the fusion hindrance.
The average trajectory, the overpassing probability and the current at the top of the barrier are plotted in Fig. 1 as a function of time for four regimes, $K=0,0<K<B_{\text {eff }}$, $K=B_{e f f}$ and $K>B_{e f f}$. Note that the time scales are different. The current is simply $j(t)=\frac{d P(t)}{d t}$. The first case corresponds to the solution of the Smoluchowski equation where inertia is neglected. The average trajectory immediately goes away from the top and the small fusion probability is only due to the diffusion. The time needed for the probability to converge is very long, about $2\left(x+\sqrt{1+x^{2}}\right) / \omega$ when $x>1$ and about $4 / \omega$ else. In the second case, when $0<K<B_{e f f}$, the average trajectory first goes in direction of the barrier top, without reaching it. The overpassing probability is still due to thermal diffusion which is very slow, but enlarged by the average motion. When the average trajectory reaches its highest point, at $t_{m}$, given by,

$\operatorname{coth}\left(\sqrt{1+x^{2}} \omega t_{m}\right)=\frac{x}{\sqrt{1+x^{2}}}+\left(1-\frac{x}{\sqrt{1+x^{2}}}\right) \sqrt{\frac{B_{e f f}}{K}}$,

the probability starts to grow. In the last case, when $K>$ $B_{e f f}$, after the average trajectory quickly passed over the barrier top, the variance is still growing and the probability is slightly decreasing. The time needed for the average trajectory to reach the top of the barrier, $t_{t o p}$, is given by, $\operatorname{coth}\left(\sqrt{1+x^{2}} \omega t_{\text {top }}\right)=\left(1+\frac{x}{\sqrt{1+x^{2}}}\right) \sqrt{\frac{K}{B_{\text {eff }}}}-\frac{x}{\sqrt{1+x^{2}}}$.

Most of the particles are in the same side of the barrier as the average trajectory. When the variance is enlarged by the spreading of the initial conditions, there are more particles on the other side of the barrier. Then the asymp- 
totic overpassing probability is larger when $K<B_{\text {eff }}$ and smaller when $K>B_{\text {eff }}$.

It is worth to mention that Fig. 1 shows that the fluxes are appreciable during a certain period of time and then vanish, which is in contrast with Kramers's process where the flux rate reaches a stationary limit. This again shows that the inverse process is a collision process with an interplay between dynamics and diffusion. It appears that the initial kinetic energy plays a major role. In Fig. 2 the final overpassing probability is plotted as a function of the initial kinetic energy, according to Eq. (11). The higher the temperature is, the smoother is the transition. When the initial kinetic energy is null or very small, temperature turns out to play the major role. This corresponds to the fusion probability calculated with a Smoluchowski type equation where fusion is solely due to diffusion. It could be seen that fusion probability increase fastly with $\mathrm{K}$ and the validity of the overdamped approximation is rather limited. This situation differs from the Kramers's problem where the overdamped approximation is valid as soon as $\beta \gg 2 \omega$. Here, the initial kinetic energy gives another strong constraint on the validity of the approximation.

Figure 2 also shows the final overpassing probability as a function of temperature when $K=0$. Applied to the fusion of heavy nuclei, this simple model shows that naturally, the fusion probability is increasing with temperature. The authors of Ref. [4] found an opposite behavior for this formation probability, solving numerically a multidimensional Langevin equation. Such a peculiar tendency would be due to the fact that the contact configuration is just on the ridge line or even slightly inside.

When $K \ll B_{\text {eff }}$, and, if, in addition, $T^{\prime}<B / 10$, conditions corresponding to Kramers's problem, the overpassing probability is extremely low and it can be expanded

$$
\bar{P}\left(t \rightarrow+\infty ; \bar{q}_{0}, \sigma_{q_{0}}, \bar{p}_{0}, T_{0}\right) \simeq \sqrt{\frac{T^{\prime}}{4 \pi \bar{B}}} \exp \left(-\frac{\bar{B}}{T^{\prime}}\right)
$$

The result has an Arrhenius type factor and could be called an "inverse Kramers's formula", considering the physical situation. However, it should be noticed that the "temperature" $T^{\prime}$ is not the one from the Langevin force, but the dynamical temperature, Eq. (12). In the specific case where $\sigma_{q_{0}}=0$ and $T_{0}=T$, one exactly gets $T^{\prime}=T$, which is more common. It should also be stressed that Kramers's formula is for the overpassing rate while the present one is for the probability.

Investigating the fusion of heavy nearly symmetric nuclei, the authors of Ref. [20] have introduced the concept of a fluctuating potential barrier in order to fit experimental data. The present results, i.e., the probability given by the error function Eq. (11) appears to be similar to the Gaussian distribution of the barrier, and tends to give a theoretical background to such an approach. The so-called fusion hindrance which corresponds to the average value of the additional fluctuating barrier could be directly evaluated with our model. Equation (13) indicates a necessity of an extra-push energy for the fusion probability to reach $1 / 2$. This additional energy, $E_{a d d}=E_{c m}-V_{B}$ with $E_{c m}$ being the center of mass available energy and $V_{B}$ the height of 
the usual barrier, is given by energy conservation:

$$
E_{a d d}=B_{\text {eff }}+\epsilon^{*}+T_{0} / 2+V_{\text {contact }}-Q-V_{B}
$$

where $\epsilon^{*}$ denotes the intrinsic excitation energy at the contact point, say $a T_{0}^{2}$. It should be emphasized that the friction at the saddle dramatically enhances the extrapush energy through $B_{\text {eff }}$. The fluctuation of the barrier should be interpreted rather as a consequence of the dissipative dynamics through the dissipation-fluctuation theorem. Here, we derived an analytical formula for the extra-push energy which could be directly applied, provided that the physical ingredients such as barrier height, nuclear viscosity... are known. A direct comparison with experimental data of the extra-push energy and the fusion probability calculated with this model, using realistic parameters, will be published in another paper [21].

Generally, for the synthesis of the superheavy elements, the incident energy should be as low as possible. In such cases, the average trajectory never reaches the saddle and fluctuations are to be taken into account to evaluate the small formation probability. We propose in this note an analytical formula for this probability as a function of the energy, Eq. (11). The connection to the dissipation in the approaching phase is done through the dispersion of the initial conditions. Nevertheless, for some cases, considering the very low fusion probability, Eq. (16) would be enough. Thus, the incident energy is largely dissipated during the approaching phase. This dissipation reduces the probability of passing over the Coulomb barrier and has to be carefully taken into consideration $[3,4]$. For some systems, when the initial kinetic energy is not completely damped out in the approaching phase, the use of a Smoluchowski type equation would appear doubtful.

The escape rate from a metastable well has been widely studied since Kramers's original paper, see Ref. [22] for a review, but not the entrance phenomena. We have then studied the inverse problem by exactly solving the Langevin equation for a simple parabolic potential barrier with dissipation and its associated fluctuations. A general solution for the overpassing probability is obtained at any time generalizing what was done in Ref. [19] and analyzed, as shown in Figs. 1 and 2. When the initial kinetic energy is completely damped out during the approaching phase, we show that the solution turns out to be a simple formula with an Arrhenius type factor that could be called "inverse Kramers's formula". It should be stressed again that even in such a case, the process is a collision one, i.e. the flux over the barrier vanishes after some times. Our results might then interest other fields of physics, especially when dissipative collisions occur. Of course, a complete theory should combine both entrance probability and escape rate. The problem depends then on the physical situation and goes far beyond the scope of this note.

We are indebted to B. Bouriquet, B.G. Giraud, G. Kosenko, C. Shen and T. Wada for interesting discussions. Two of us (Y.A. and J.D.B.) thank GANIL for its warm hospitality and support. One of us (D.B.) thanks JSPS for its support and the Yukawa Institute for its warm hospitality. This work is partially supported by the Grant-in-Aids $n^{\circ} 13640278$ from JSPS. 


\section{References}

1. S. Hofmann, Rep. Prog. Phys. 61, 210, (1996)

2. P. Armbruster, Annu. Rev. Nucl. Part. Sci. 50, 411 (2000)

3. G. Kosenko, C. Shen and Y. Abe, J. Nucl. Radiochem. Sci. 3, $19(2002)$

4. C. Shen, G. Kosenko and Y. Abe, Phys. Rev. C66, $061602(\mathrm{R})(2002)$

5. C.E. Aguiar, V.C. Barbosa, D. Donangelo and S.R. Souza, Nucl. Phys. A491, 301 (1989); C.E Aguiar, V.C. Barbosa and D. Donangelo, Nucl. Phys. A517, 205 (1990)

6. Y. Abe, Eur. Phys. J. A13, 143 (2002); Y. Abe, D. Boilley, G. Kosenko, J.D. Bao, C.W. Shen, B. Giraud and T. Wada, Prog. Theor. Phys. Suppl. 146, 104 (2002); Y. Abe, D. Boilley, B. Giraud, G. Kosenko and C. Shen, to appear in Heavy Ion Phys. (Hungary); Y. Abe, D. Boilley, G. Kosenko and C. Shen, to appear in Acta Physica Polonica B.

7. G.G. Adamian, N.V. Antonenko, W. Scheid and V.V. Volkov, Nucl. Phys. A627, 321 (1997)

8. Y. Abe, Y. Aritomo, T. Wada and M. Ohta, J. Phys. G: Nucl. Part. Phys. 23, 1275 (1997); Y. Aritomo, T. Wada, M. Ohta, and Y. Abe, Phys. Rev. C 59, 796 (1999)

9. W.J. Swiatecki, K. Siwek-Wilczynska and J. Wilczynski, to appear in Acta Physica Polonica B

10. Y. Abe, S. Ayik, P.-G. Reinhard and E. Suraud, Phys. Rep. 275, 49 (1996)

11. P. Fröbrich and I.I. Gontchar, Phys. Rep. 292, 131 (1998)

12. H. A. Kramers, Physica VII, 4, 284 (1940)

13. J.D. Bao and D. Boilley, Nucl. Phys. A707, 47 (2002)

14. D. Boilley et al, in preparation

15. Y. Abe, D. Boilley, B.G. Giraud and T. Wada, Phys. Rev. E61, 1125 (2000)
16. G.E. Uhlenbeck and L.S. Ornstein, Phys. Rev. 36, 823 (1930)

17. S. Chandrasekhar, Rev. Mod. Phys. 15, 1 (1943)

18. H. Hofmann and R. Samhammer, Z. Phys. A322, 157 $(1985)$

19. H. Hofmann and P.J. Siemens, Phys. Lett. 58B, 417 (1975)

20. A.B. Quint, W. Reisdorf, P. Armbruster, F.P. Heßberger, S. Hofmann, J. Keller, G. Müzenberg, H. Stelzer, H.-G. Clerc, W. Morawek and C.-C. Sahm, Zeit. Phys. A346, 199 (1993)

21. J.D. Bao, D. Boilley and Y. Abe, in preparation

22. P. Hänggi, P. Talkner and M. Borkovec, Rev. Mod. Phys. 62, $251(1990)$ 
Fig. 1. Average trajectory, overpassing probability and current at the top of the barrier as a function of time for the four regimes, $K=0$, (first column) $K=B_{e f f} / 2$ (second column), $K=B_{e f f}$ (third column) and $K=2 B_{\text {eff }}$ (last column). For each case, two temperatures were chosen, $T=B / 5$ (solid line) and $T=B / 2$ (dashed line). These are non-dimensional plots. Here, $x=\frac{\beta}{2 \omega}=3, T_{0}=0$ and $\sigma_{q_{0}}=0$. Note that each column has a different time scale.

Fig. 2. Left: Final overpassing probability as a function of the initial kinetic energy for two temperatures, $T=B / 10$ (solid line) and $T=B / 2$ (dashed line). Right: Final overpassing probability as a function of the temperature when the initial kinetic energy $K=0$. Here, $x=\frac{\beta}{2 \omega}=3, T_{0}=0$ and $\sigma_{q_{0}}=0$. 

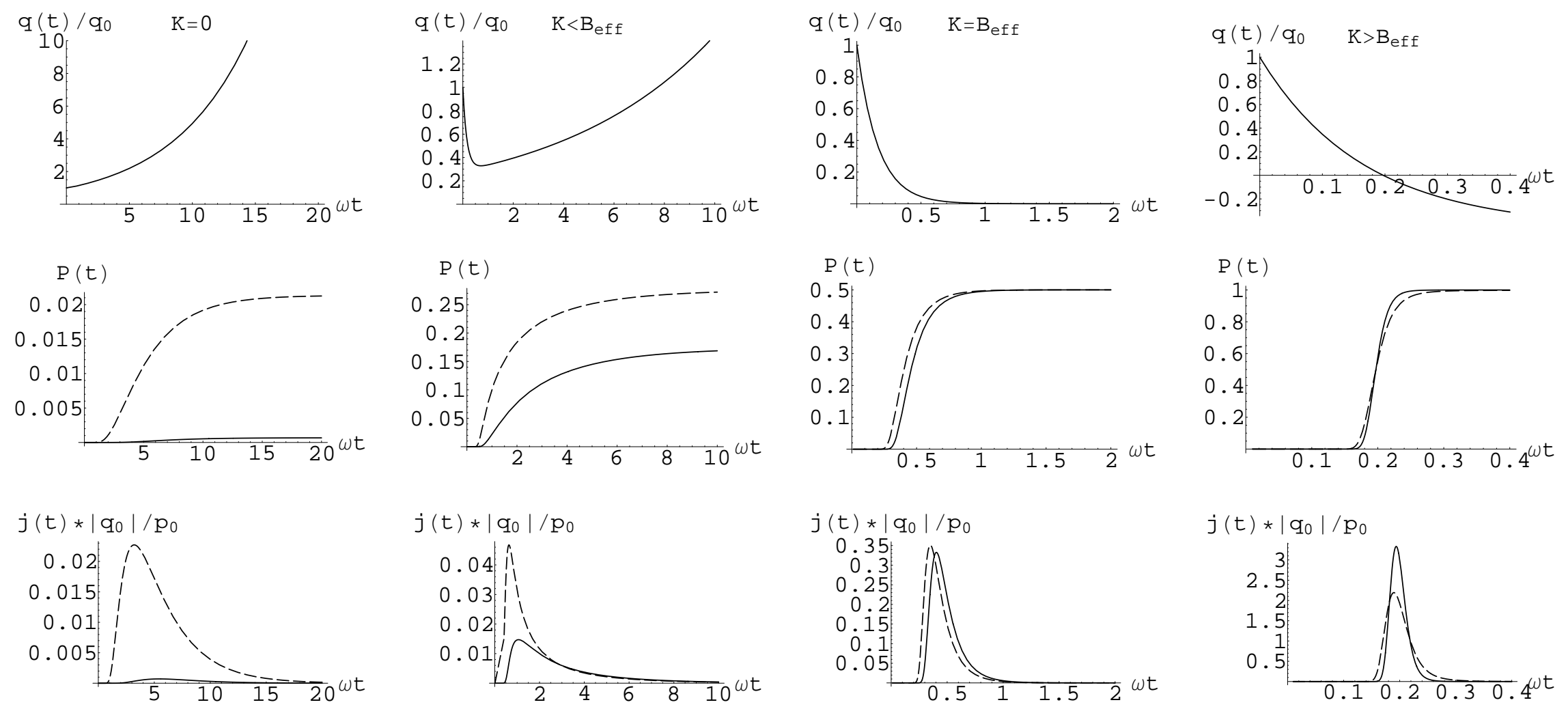


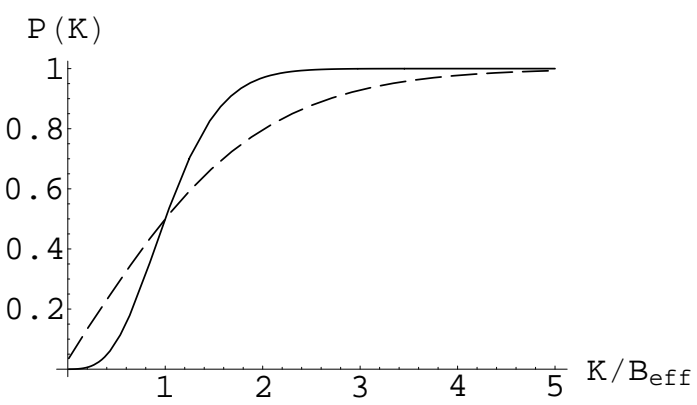

$\mathrm{P}(\mathrm{T})$

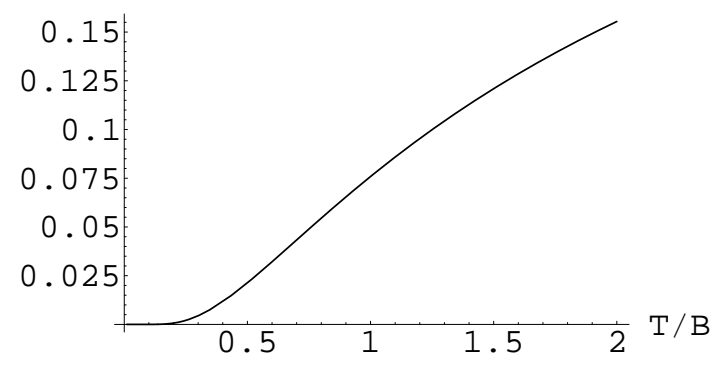

